\title{
Perspective
}

\section{Lichens and microbial syntrophies offer models for an interdependent route to multicellularity}

\author{
Eric Libby ${ }^{1}$ (D) and William C. Ratcliff ${ }^{2}$ \\ ${ }^{1}$ Department of Mathematics and Mathematical Statistics, Integrated Science Lab (Icelab), Umeå University, Umeå 90187, Sweden and ${ }^{2}$ Department of Biological \\ Sciences, Georgia Institute of Technology, 310 Ferst Drive NW, Atlanta, GA 30332, USA
}

\begin{abstract}
The evolution of multicellularity paved the way for significant increases in biological complexity. Although multicellularity has evolved many times independently, we know relatively little about its origins. Directed evolution is a promising approach to studying early steps in this major transition, but current experimental systems have examined only a subset of the possible evolutionary routes to multicellularity. Here we consider egalitarian routes to multicellularity, in which unrelated unicellular organisms evolve to become a multicellular organism. Inspired by microbial syntrophies and lichens, we outline three such routes from a system of different species to an interdependent relationship that replicates. We compare these routes to contemporary experimental systems and consider how physical structure, the threat of invasion, division of labour and co-transmission affect their evolution.
\end{abstract}

Key words: division of labour, experimental evolution, major transitions, microbes, multicellularity, syntrophy

(Accepted 25 February 2021)

\section{Models to Study the Evolutionary Origins of Multicellularity}

The evolution of multicellular organisms from unicellular ancestors is one of the major transitions of evolution (Maynard Smith \& Szathmáry 1995). It paved the way for new kinds of organisms that were more complex than their unicellular ancestors in terms of scale, organization, behaviours and life cycles. While multicellularity has evolved dozens of times independently (Grosberg \& Strathmann 2007), much remains to be learned about the early steps of this major evolutionary transition. Much of what we know has been inferred from limited geological and fossilized remains (Knoll 2011), or through phylogenetically-informed comparisons among living organisms (King 2004; Suga et al. 2013; Nagy et al. 2018). Importantly, we lack basic knowledge about the diversity of possible routes to multicellularity and how increased complexity arises de novo.

In the last decade, laboratory model systems have provided some insight into how simple multicellularity can evolve. These systems rely on experimental evolution techniques that subject single-celled microbes to a selective regime that promotes the evolution of replicating groups of cells. While replicating groups of cells are a far cry from a fully-fledged multicellular organism, this is usually considered a necessary first step. One model system produced by this approach is snowflake yeast, which evolved from unicellular Baker's yeast (Ratcliff et al. 2012) in response to artificial selection for rapid settling through liquid growth media.

Author for correspondence: Eric Libby. E-mail: elibbyscience@gmail.com

Cite this article: Libby E and Ratcliff WC (2021) Lichens and microbial syntrophies offer models for an interdependent route to multicellularity. Lichenologist 53, 283-290. https://doi.org/10.1017/S0024282921000256
Since clusters of cells settle more quickly than solitary cells, this selective regime leads to the evolution of yeast mutants that do not separate after reproduction. The genetics underlying this change are remarkably simple and repeatedly involve a single gene, ACE2, which plays a key role in regulating mother-daughter cell separation after mitosis (Ratcliff et al. 2015; Tan et al. 2019). Loss of function mutations in this gene produce yeast that grow as fractal-like trees of connected cells, with a highly conserved topology (Ratcliff et al. 2015).

Reproduction in snowflake clusters occurs as a natural consequence of growth (see Fig. 1A). As snowflake clusters grow, they run out of space in the central parts of the group, leading to the accumulation of cell-cell strain and eventually group fracture (Libby et al. 2014; Jacobeen et al. 2018a,b). Group fracture provides a key attribute: it allows groups to reproduce, propagating themselves by severing branches, which themselves grow up to their parent's size before fracturing. This process allows groups to function as units of selection, surviving or dying as a unit depending on whether they can settle to the bottom of a test tube quickly enough. Continued evolution in this experiment has seen novel group-level traits arise through changes in celllevel traits which affect the way that cells interact within the group. For example, groups evolve to be considerably larger by evolving more elongate cells, which generates more room for cellular movement within the cluster and reduces strain-based fragmentation (Jacobeen et al. 2018a, b).

Similar experimental approaches selecting for larger groups have led other unicellular organisms to evolve multicellularity. For example, experimental evolution of Chlamydomonas reinhardtii, a unicellular relative of the multicellular volvocine green algae, under selection for rapid sedimentation resulted in the 


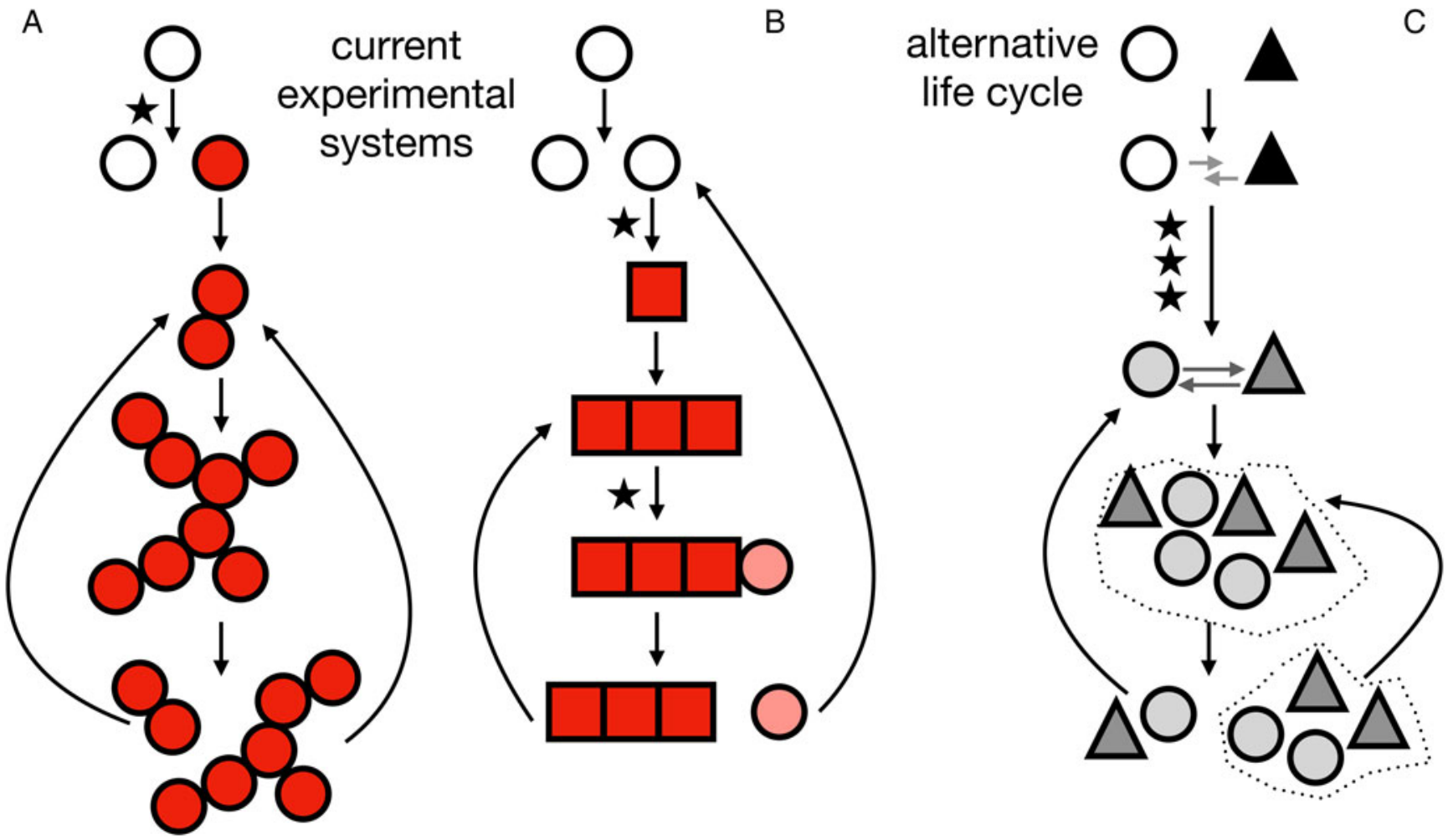

Fig. 1. Life cycles at the origins of multicellularity. A, life cycle corresponding to the snowflake yeast. A mutation (indicated by a star) results in a mutant (indicated by red/filled circles) that cannot separate from its daughter cells. As cells reproduce the group increases in size until fragmentation results in two daughter groups that then repeat the life cycle. B, life cycle corresponding to the smooth-wrinkly system of Pseudomonas fluorescens. A mutation causes a phenotypic switch (indicated by a different, square-shaped cell). The group grows until another mutation recapitulates a phenotypic state similar to the unicellular ancestor, completing the life cycle. $\mathrm{C}$, an example of an alternative multicellular life cycle not explored in current experimental systems. It begins with two different species (indicated by a white circle and a black triangle). An initial interaction (indicated by grey arrows) evolves through several mutations (indicated by stars) to become a more interdependent, possibly regulated, relationship. The multicellular life cycle is then repeated through population expansions and dispersal events (the dotted lines represent environmental patches). In colour online.

evolution of multicellular groups (Ratcliff et al. 2013). Chlamydomonas reinhardtii evolved a similar life cycle in a different experiment where it was exposed to size selection, this time via the presence of a gape-limited predator (Herron et al. 2019). As with snowflake yeast, the groups grew until physical strain caused them to fracture into smaller groups. The repeated evolution of multicellular life cycles that rely on stress-mediated fragmentation for reproduction across experimental systems probably reflects common features of the experiments, including clonal population growth and a population bottleneck imposed through size selection.

A qualitatively different experimental system for studying multicellularity uses the SBW25 strain of the soil bacterium Pseudomonas fluorescens (see Fig. 1B). In this system, the experimental protocol exploits a phenotypic switch that regularly evolves when SBW25 is cultured in liquid media (Rainey \& Travisano 1998; Rainey \& Rainey 2003). Initially, SBW25 expresses a unicellular phenotype that is characterized by the smooth colonies it forms when grown on plates. As a consequence of rapid reproduction, the 'smooth' cells reliably produce mutants, called 'wrinkly' cells. The wrinkly cells have a mutation, typically in one of three pathways, that disrupts regulation of the wss operon (Lind et al. 2015). Dysregulation of the wss operon causes wrinkly cells to overproduce extracellular cellulose and stick to their offspring, forming mats. The expanding population of smooth and wrinkly cells consume the oxygen in the media within a day and the only available oxygen is at the air-liquid interface (Koza et al. 2011). Due to their mat formation, the wrinkly types colonize this niche and benefit from access to oxygen. As the wrinkly cells reproduce, they give rise to new mutants that do not produce cellulose and, thus, resemble the ancestral smooth phenotype. After a few days, the mat collapses and releases these new smooth mutants into the broth, thereby completing a life cycle. The new smooth mutants can then be cultured in fresh media to repeat the process.

The Pseudomonas fluorescens system demonstrates how a multicellular life cycle can emerge by exploiting pre-existing traits. Many unicellular organisms make adhesive polymers to interact with each other and their environment (Dunne 2002). It seems plausible that modification of these adhesive molecules can lead to the formation of groups of cells, and then subsequent modifications can produce single cells. Although relying on mutations to switch adhesive molecule production on and off may be cumbersome, it is also possible for an epigenetic mechanism to evolve. Again, the Pseudomonas fluorescens system illustrates how this might occur. Clonal populations of P. fluorescens, the SBW25 strain in particular, can exhibit cell to cell variability in the production of adhesive polymers (Gallie et al. 2015). The underlying variability might be accidental or adaptive, potentially acting as a bet-hedging trait. Regardless, mutations can enhance this stochastic phenotypic variation to generate mixed populations of cells: those producing significant quantities of adhesive molecules and 
those not making any (Gallie et al. 2015). Lineages of cells can then switch between making adhesive molecules or not, giving rise to a phenotypic switch similar to one found in the smoothwrinkly life cycle without the need for mutations. Thus, the evolution of multicellularity might harness unicellular preadaptations or survival strategies and modify their frequency to reliably produce replicating groups of cells.

In concert with these experimental approaches, theoretical work has sought to extract general principles underlying the transition to multicellularity. As in the experimental approaches, theoretical models typically focus on simple replicating groups of cells (Roze \& Michod 2001; Gavrilets 2010; Libby \& Rainey 2013a; Tarnita et al. 2013) and consider the various ways they can originate, persist, or evolve. Models that investigate the origination of multicellularity often identify environmental or ecological conditions that select for the formation of groups, such as avoiding predation (Boraas et al. 1998; Herron et al. 2019), surviving stressful conditions (Smukalla et al. 2008), gaining metabolic advantages (Koschwanez et al. 2011), or colonizing new niches (Bonner 2009). Models that investigate the persistence of multicellularity typically determine traits or conditions that prevent groups of cells from reverting to unicellular forms, for example ratcheting traits that confer fitness benefits to groups but fitness costs to single cells (Libby \& Ratcliff 2014; Libby et al. 2016). Lastly, models that investigate the evolution of early multicellularity explore how aspects of the group or its life cycle affect the rate of adaptation or what novel traits emerge (Roze \& Michod 2001; Willensdorfer 2009; Ratcliff et al. 2017). Together these models have revealed many insights, especially concerning the variety of paths to evolving multicellularity and the significant ways in which these paths dictate future evolution.

As a result of this theoretical and experimental work, a consensus of the evolutionary dynamics driving the origin of simple multicellularity has emerged. First, single-celled organisms must evolve into simple multicellular groups. Then, these multicellular groups must become 'units of selection' that are capable of reproduction and evolution by selection, acquiring multicellular or 'group-level' adaptations. For example, groups may evolve a reproductive division of labour among constituent cells such that there are somatic and germ cells. Importantly, the group-level adaptations must resist cell-level adaptations, such as cancer, over evolutionary timescales (Queller \& Strassmann 2009). Finally, the group of cells becomes an evolutionary individual in its own right when its fitness is more than an additive function, for example average, of the fitnesses of its constituent cells (Michod 2005; Okasha 2006).

\section{Alternative Evolutionary Routes to Multicellularity}

There is an important caveat to the experimental and theoretical work discussed: it explores a particular set of paths to multicellularity in which the initial transition between unicellularity and multicellularity is mediated by mutations that directly result in the formation of multicellular groups. In snowflake yeast, loss of function in the ACE2 transcription factor causes a unicellular yeast to grow as a multicellular group (Ratcliff et al. 2015). In the smooth-wrinkly $P$. fluorescens system the mutation could occur in any of the pathways regulating expression of the wss operon, though they repeatedly occur more often in a particular negative regulator wspF (Bantinaki et al. 2007; Lind et al. 2015). While the specific genes required for multicellular growth in experimentally evolved Chlamydomonas are not well resolved, mutations were over-represented in cell cycle and reproductive processes, and in volvocine-specific genes (Herron et al. 2018). For snowflake yeast and mat-forming Pseudomonas, multicellular and unicellular forms can be transformed into each other through altering just one gene in one cell. This transmutability makes these systems useful for controlled scientific studies, but it may not be a hallmark of all evolutionary routes to multicellularity.

To identify possible alternative paths to multicellularity, we first consider what is required for the evolution of replicating groups of cells. In particular, we note that there are three basic requirements: 1 ) there needs to be more than one cell involved, 2) the collection of cells needs to form something with a group structure, and 3) the group needs a way of replicating (Libby \& Rainey 2013a). Of the three requirements, the second perhaps needs clarification concerning its role. In essence, the structure of a group acts to distinguish primitive multicellularity from an arbitrary collection of cells. It ensures that there is a distinction between members and non-members of a group, by requiring some connection, either direct or indirect, between cells in the same group. Cells can then share the benefits of being in the same group and contribute to its survival along with any emergent group-level traits. If we consider these three requirements within the snowflake yeast system, we see that all three are provided by a mutation in ACE2. By causing cells to stay attached following reproduction, the mutation connects all cells in a group and causes them to experience the same evolutionary fate: cells live or die if their groups live or die. Since the connection between cells can break under strain, there is also a mechanism for group reproduction. If we turn to the $P$. fluorescens experimental model, we see that the initial mutation that generates a wrinkly type satisfies requirements 1 and 2 but does not provide a mechanism for group replication; that has to come from a second mutation which restores the original phenotype. However, the abundance of paths between smooth and wrinkly types allows all three requirements to be met (Lind et al. 2015).

Starting with a single unicellular species, it is difficult to envision an evolutionary path to multicellularity that does not first involve a mutation leading to the formation of groups, satisfying requirements 1 and 2 . Thus, we consider an alternative possibility in which a group of cells does not evolve from a single unicellular species but through the interaction of at least two different species. In this scenario, the first requirement is immediately satisfied. The second and third requirements imply that the community of cells must evolve a group structure and some way of propagating the community (i.e. a mode of replication) (see Fig. 1C). Indeed, selection on interacting unicellular organisms has been considered a path for 'egalitarian' transitions (Queller 1997) to multicellularity, in which unrelated unicellular organisms come together to form a new kind of multicellular organism (Pande et al. 2014; Szathmáry 2015; Queller \& Strassmann 2016). In a sense, the origin of eukaryotic cells from a symbiosis between an archaeal host and an endosymbiotic alphaproteobacterium (Zaremba-Niedzwiedzka et al. 2017) is a form of egalitarian multicellularity.

One challenge in considering a path to multicellularity that starts with two different species is determining the initial type of interaction between the species. There are many possible initial population structures involving two different species, and it is possible that some are much more likely than others to yield a form of multicellularity. To limit the scope, we draw inspiration from two examples of multi-species systems with evolvable grouplevel phenotypes: microbial syntrophies and lichens. We note that 
neither is a canonical multicellular organism and there are probably key aspects of their particular evolution that is sui generis. Nonetheless, we can adopt a theoretical approach and use general features of their evolution to explore the potential trajectories from a collection of different unicellular species to a type of simple multicellularity (i.e. replicating groups of cells).

\section{Lichens and Microbial Syntrophies as Higher-level Units}

A defining trait of both microbial syntrophies and lichens is an interdependent relationship between different species. In microbial syntrophies, relationships are typically mediated by the production or exchange of biochemical compounds or metabolites (Stams \& Plugge 2009; Morris et al. 2013). For instance, methanogenic archaea and fermenting bacteria have a syntrophic relationship that allows them to survive the harsh conditions of hydrothermal vents (Schink 2005). The archaea consume the hydrogen waste of fermenting bacteria which would otherwise accumulate and inhibit their ability to ferment. The pair of microbes have complementary metabolisms such that through the exchange of compounds they can survive together in an environment where they would struggle in isolation. In comparison to microbial syntrophies, lichen relationships are more complex and vary between partners and species (Hawksworth 1988; Armaleo \& Clerc 1991; Richardson 1999; Grube \& Berg 2009; Spribille et al. 2016; Tuovinen et al. 2019); however, the canonical lichen symbiosis features an interdependent relationship between a fungus and a photobiont in which the photobiont provides energy from photosynthesis and the fungus provides protection and nutrients (Nash 2008). Similar to the example of archaea and fermenting bacteria, the lichen can survive and persist in environments that would challenge its constituent members in isolation (de la Torre et al. 2010).

The interdependent relationship found in microbial syntrophies and lichens produces a group phenotype in which the whole is more than a sum of its parts. A distinction between a group phenotype and the phenotype of its constituent parts is prevalent in studies of multicellularity (Okasha 2006) where it provides contrast between a population of cells and a multicellular group of cells (Queller \& Strassmann 2009). For lichens, one key emergent group phenotype is a macroscale thallus structure that facilitates the symbiosis (Honegger 1998; Nash 2008). Neither the photobiont nor the mycobiont builds it without the other. Its large scale also leads other organisms to interact with the lichen as a whole differently than they would either constituent organism; for example reindeer graze on lichens but not on isolated populations of mycobionts or photobionts. For microbial syntrophies, the group phenotype is represented by its growth or survival in different metabolic environments. In both cases a division of labour gives rise to a group phenotype in which changes in cell traits can modify the group phenotype. For example, if one species in a syntrophy has a loss of function mutation inhibiting its production of a certain metabolite, then the syntrophy can collapse (Oliveira et al. 2014; Kallus et al. 2017). Thus, there is the potential for group phenotypes to evolve in response to cellular mutations and possibly adapt.

A central challenge that determines whether a community can act as a higher-level of selection is whether it can replicate, that is whether groups can reproduce. This challenge is particularly pertinent for multi-species communities such as lichens or microbial syntrophies because, in order for the group to reproduce, at least one cell of each species should be present in the group's 'offspring'
(Oliveira et al. 2014; Estrela et al. 2016; Queller \& Strassmann 2016). In general, there seem to be two main categories of solution to this problem: groups can reproduce through fragmentation or through a process of dispersal and reassembly. With fragmentation, a part of the group breaks off from the original group and is able to grow and reproduce similar to the parent (Highsmith 1982). In snowflake yeast this occurs naturally because of the fractal nature of the growth form. For multi-species communities, as long as a group splits in such a way that both fragments contain the necessary species, then this mode of reproduction should allow for group reproduction. This mode of reproduction is a common form of asexual propagation in lichens (Bowler \& Rundel 1975) and may occur in microbial syntrophies, though it is difficult to verify in natural populations. The second form of group reproduction involves a dissociation between some cells in the multi-species community and a re-establishment of the relationship elsewhere. In lichens, this typically occurs through the production of fungal spores that are released from the lichen and re-associate with free-living photobionts. For microbial syntrophies, a similar life cycle has been proposed through a process of random dispersal into new habitats (Cremer et al. 2012; Oliveira et al. 2014). In both cases the cells that leave the original group and re-establish a new group must contain in their genome traits for perpetuating the interdependent relationship and the emergent group phenotype.

Although lichens and microbial syntrophies may function as units of selection, they differ from a fully-fledged multicellular organism and even the primitive multicellularity exhibited by current experimental systems such as snowflake yeast. The differences between these systems reflect variations in their 'organismality', a term highlighting the ambiguity in the definition of an organism (Queller \& Strassmann 2009). Biology has terms that are often used heuristically without precise, universally accepted definitions, such as the concept of 'species' (Hey 2001) or 'fitness' (Ariew \& Lewontin 2004). The study of major evolutionary transitions, such as multicellularity, in particular depends on how we define 'organisms'. A central concept to emerge from philosophers studying biological individuality is that whether an entity is an organism is not binary, but rather lies on a spectrum (Godfrey-Smith 2009; Queller \& Strassmann 2009). For example, Queller \& Strassmann (2009) mapped groups of two species in terms of cooperation and cellular conflict and determined a lichen to be more organismal than a system of squid and its Vibrio fischeri, and less organismal than the system of a eukaryotic cell and its mitochondria. The same axes were also used to relate different types of multi-celled populations and multicellular populations. It is likely that some transitions from unicellularity to multicellularity will appear to be rapid and unambiguous, resulting in an entity that is much more organismal than its ancestors within a single generation, while other transitions may never have an easily identifiable moment of transition. For example, the evolution of snowflake yeast has a distinct transition arising from a single causal mutation in which a well-defined group emerges from a unicellular ancestor. In contrast, a microbial syntrophy may always appear as a hybrid between a form of sociality and something organismal, stuck between the two evolutionary states of uni- and multi-cellularity. We assume that the type of multicellularity that evolves from a collection of two unicellular species (e.g. a form resembling a microbial syntrophy or the lichen symbiosis) may be a higher-level unit just not to the same degree as a plant or animal. Importantly, we do not argue that lichens or microbial syntrophies are multicellular organisms but rather 
they can serve as models to inform our understanding of the evolution of multicellularity.

\section{Evolutionary Origins}

If we use microbial syntrophies and lichens as guides to understanding how multicellularity might evolve from a collection of unicellular species, then it is necessary to consider how a collection of cells might evolve an interdependent relationship capable of evolving group-level adaptations. One simple possibility is serendipity: two originally self-reliant cells somehow end up in an environment in which neither can survive on their own, but the pair can survive together (see Fig. 2A). The pair's survival allows them to colonize new, similar environments and propagate the relationship. In this case, it would be difficult to isolate any causal mutation for the interdependent relationship. It might be possible to identify mutations that can disrupt the relationship but its origination is a product of fortuitous complementary evolution. Interestingly, computational work has shown that many microbes, with no known history of interaction, can be paired in environments in which they survive through the exchange of metabolites (Klitgord \& Segrè 2010). Metabolic complementarity has also been found in pairings of random, computer-generated metabolisms in the absence of any evolutionary history (Libby et al. 2019). Together, these studies suggest that the serendipitous path to syntrophy (and possibly multicellularity) does not need specific prior adaptations and may be more likely to occur than expected.

An alternative evolutionary path to the type of metabolic interdependence found in syntrophy requires that at least one of the microbes be initially self-reliant and then the mutual dependence is a derived, evolved state (see Fig. 2B). In this scenario, the mutual dependence is usually posited to occur through deleterious mutations that erode metabolic independence but free microbes of the burden of producing costly metabolites (Morris et al. 2012; Hillesland et al. 2014; D'Souza \& Kost 2016). The Black Queen Hypothesis (Morris et al. 2012) extends this scenario to describe how a group of independent cells can evolve into a community in which metabolic functions are partitioned across different, interdependent cells.

Finally, lichens illuminate another way to evolve an interdependent relationship between two species that lies somewhere between the serendipitous and Black Queen paths. We focus on the simplest abstracted form of a lichen that features an interdependent relationship between a fungus and a photobiont; many modern lichens have other partners (Grube \& Berg 2009; Spribille et al. 2016), but these were probably later additions following evolution of the initial pairwise relationship. Phylogenetic analyses suggest that the initial relationship of the fungus and photobiont could have ranged from mutualistic to parasitic (Gargas et al. 1995). Experiments co-culturing a fungus and photobiont have also shown that a mutualistic relationship may have occurred serendipitously due to a simple change in the environment (Hom \& Murray 2014). Here, we consider an initial parasitic relationship because it is a novel starting state for studies of the evolution of multicellularity. Although the relationship between fungi and their photobionts seems to be fluid, with the potential to evolve between parasitism and mutualism (Gargas et al. 1995; Lutzoni et al. 2001), there is a lack of experimental or observational data on the mechanisms or conditions that allow this to occur. Instead, we draw insights from recent experimental systems using bacteria and phages (Poullain et al. 2008;
Shapiro \& Turner 2018) and outline a possible path from parasitism to an interdependent relationship, for example lichen symbiosis (see Fig. 2C).

At the start the interaction would not have been interdependent, the fungus would simply be an opportunistic parasite of the photobiont. From there it could evolve along two basic lines: i) the fungus could remain opportunistically parasitic of the photobiont, or ii) it could evolve a more specialized parasitic relationship (i.e. increase its host specificity). In the first case, the interaction between the two would remain transient; by contrast, the second case could lead to more sustained interactions. If the fungus evolved to preferentially parasitize the photobiont then mutations that increased the likelihood of interactions between the two would be selected for in fungal populations; note the process could also happen in reverse whereby more frequent interactions between the two species promote fungal populations to evolve a more specialized parasitic relationship. Either case would lead to repeated interactions between fungi and photobionts and thus perpetuate the parasitism across future generations. By evolving ways to perpetuate interactions with the photobiont, the fungus effectively evolves a form of group reproduction, ensuring propagation of fungus-photobiont groups. At this point, the fungus need not provide any benefit to the photobiont and the relationship could always be one of parasitism. Yet, depending on the environmental conditions affecting the life history of fungus-photobiont groups, there could be selective pressure for the fungus to evolve restraint or invest in the growth of the photobiont to ensure successful transmission/reproduction, similar to other host-parasite relationships (Kochin et al. 2010). If this were to occur, then it would facilitate the accumulation of mutations that regulate their interactions, producing more long-lived lichens that feature interdependent relationships, if only transiently.

\section{Pathway Features and Challenges}

In the previous section, we considered a set of paths to multicellularity starting with a group of different species that evolves an interdependent relationship and a way of propagating or transmitting that relationship across generations. The paths differ in terms of when and how co-transmission and interdependence evolve, causing them to face unique challenges. For example, in the serendipitous path the interdependent relationship between species is present at the start but co-transmission of the two species must evolve for there to be some form of reliable group reproduction. In contrast, the parasitic path may start with some form of co-transmission, assuming that the parasite is successful, and then interdependence must evolve for the relationship to be cooperative and more like a multicellular organism. Despite the variation among paths, they share characteristic features that differ with current experimental and theoretical models of the evolution of multicellularity.

A key area of difference between the novel paths to multicellularity and currently studied paths is the structure of groups. In models where multicellularity evolves from a single species, called a 'fraternal' transition (Queller 1997), the formation of groups usually involves a physical process of staying together (Tarnita et al. 2013) that creates well-defined, clonal groups. For example, in snowflake yeast cells remain together following reproduction so groups are a connected set of cells, and in P. fluorescens cells produce an extracellular glue and stick to their offspring. In contrast, the novel paths to multicellularity described above have a group structure that is defined by interdependent relationships. Species 
A

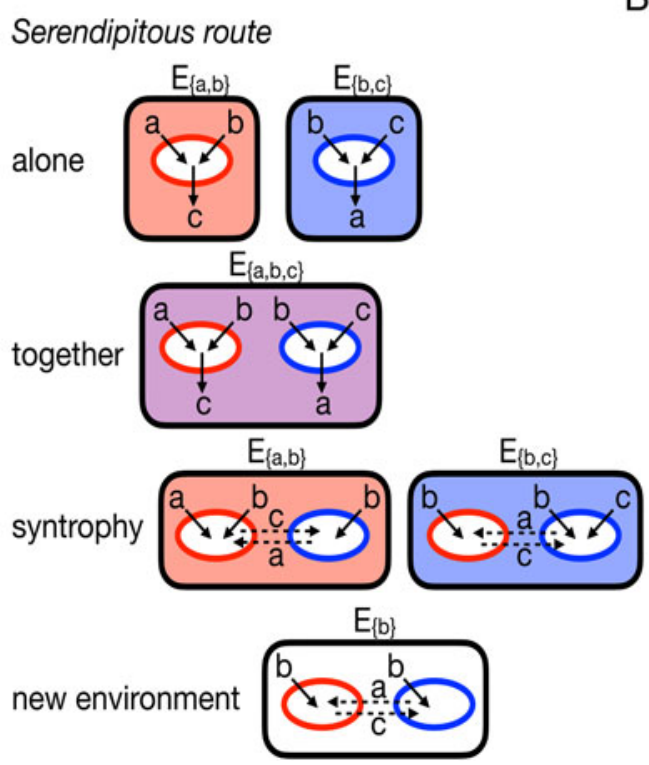

B

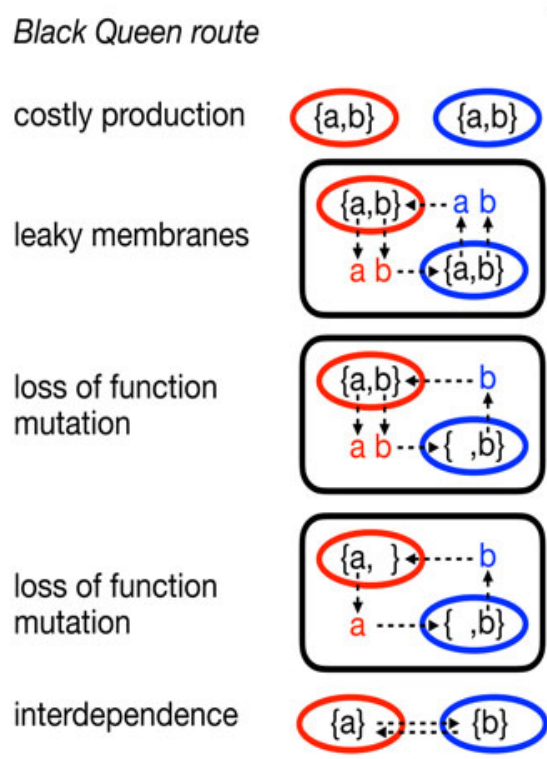

C Parasitism route
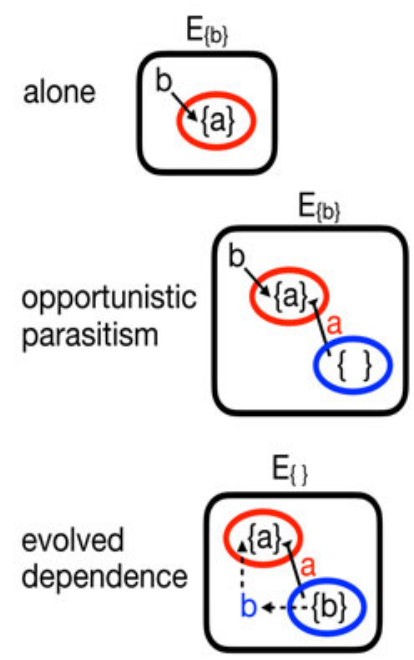

Fig. 2. Alternative, egalitarian routes to evolving multicellularity. A, the serendipitous route starts with two organisms (coloured red and blue) capable of surviving in environments that provide them with the necessary metabolites, $E_{a, b}$ provides metabolites $a$ and $b$ and $E_{b, c}$ provides metabolites $b$ and $c$. Each organism produces waste products they do not need, the red organism excretes $c$ and the blue organism excretes $a$. Should the organisms share an environment, say $E_{a, b, c}$, they can then engage in a syntrophy that allows them to survive in environments where one or both could not survive before. B, the Black Queen route begins with two self-sufficient organisms that produce the necessary, yet energetically costly, metabolites $a$ and $b$. It is assumed that they have some permeability in their membranes such that some of their metabolites diffuse out into the environment and become available to others. The availability of metabolites in the environment allows for mutations to arise in the population that disable the production of metabolites. Ultimately the organisms become reliant on each other: the blue organism needs $a$ from the red and the red needs $b$ from the blue. $C$, the parasitism route starts with an organism (red) that takes in a product $b$ from the environment and produces $a$. An opportunistic parasite (blue) arrives and steals $a$ from the red organism. In response to a selective pressure, such as another competing parasite or changing environments or improved growth, the parasite evolves to provide a benefit to the red organism.

are not necessarily physically connected by a glue or membrane but rather by the functional reliance on each other. Certainly they can evolve physical connectedness, for example a lichen thallus, but at the onset it is not strictly necessary and it may never evolve (Queller \& Strassmann 2016).

The lack of a connected group structure in the novel paths to multicellularity can make groups permeable so that members might leave and others might join. While some permeability is necessary for groups to reproduce (either via fragmentation or dispersal/reassembly), it also allows for new organisms, not originally part of the group, to invade and possibly alter existing relationships. The outcome of invasion and population mixing is likely to vary depending on the context, but studies of the evolution of microbial cooperation have shown that well-mixed, fluid environments can promote the rise of evolutionary cheats that disrupt cooperative relationships (Nadell et al. 2016). However, there is also evidence that some interdependent metabolic relationships can be maintained and withstand invasion from cheating types (Pande et al. 2014). If we turn to lichens, which have more physical structure than most microbial syntrophies, there is a large class of lichenicolous fungi which parasitizes existing lichen symbioses and sometimes steals photobionts (Richardson 1999; Nash 2008). However, invasions can also lead to novel forms of cooperation. For example, some lichens are found to be a symbiosis made up of three partners which suggests other partners can become integrated into the group (Spribille et al. 2016; Tuovinen et al. 2019). A similar process would probably be more difficult in snowflake yeast and Pseudomonas fluorescens because they reproduce groups through single-cell bottlenecks which would inhibit transmission of any invaders to future generations.

A salient feature of these novel routes to multicellularity is their division of labour. In all of the novel routes division of labour is present either at the start or within the first mutation; group structure relies on the interdependence that comes from division of labour. Moreover, groups benefit from being able to draw upon the functional complexity and evolutionary history of different genomes. In contrast, current model systems for multicellularity may not necessarily exhibit any division of labour. For example, snowflake yeast are clonal groups with all cells initially expressing a similar phenotype. Evolving division of labour would be a later, possible group-level adaptation and a step towards greater multicellular complexity. The case is different for Pseudomonas fluorescens because division of labour between the mat-forming wrinkly types and the free-living smooth types is essential for group reproduction. The problem is that it relies on a ready supply of mutations to switch back and forth and experimental evidence suggests that such mutations may be increasingly less available over many life cycles (McDonald et al. 2009; Lind et al. 2015). Certainly, organisms can circumvent this mutational supply problem by evolving epigenetic ways of dividing labour such as a phenotypic switch or regulation (Libby \& Rainey 2013b; Gallie et al. 2015), but it requires multiple iterations of the life cycle to fix in a population. This highlights a difficulty with evolving division of labour in clonal experimental systems: it requires mutations that cause temporal or spatial phenotypic differentiation. In contrast, egalitarian routes to multicellularity capitalize on phenotypic differences between cells, 
leveraging the benefits of cellular differentiation as an initial driver of multicellular evolution.

Finally, we consider a central challenge in the egalitarian routes to multicellularity: reproduction. In either the fragmentation or the dispersal/reassembly mode of reproduction, two species must ensure that their relationship persists in order to propagate the nascent multicellular organism. The more interdependence among the species, that is the cells of the nascent multicellular organism, the more essential it is to ensure that they co-transmit. Work by Oliveira et al. (2014) showed that uncertainty in co-transmitting with a syntrophic partner can limit the evolution of metabolic interdependence. Without a way of ensuring co-transmission, species must balance between specificity and generality; greater specificity could lead to greater mutual gains from a partnership but at the cost of lower survival in its absence. One way to navigate uncertainty in co-transmission while not being completely self-reliant is for species to evolve reliance but not on specific partner species, a form of general dependence but not specific interdependence. Indeed, there is evidence that metabolism in bacteria is a property of a community of bacteria, partitioned across potentially many different species (D'Souza et al. 2018). Yet despite the challenge of co-transmission, lineages of lichens have found ways to reliably reproduce using a dispersal/reassembly life cycle (Nash 2008) and maintain specificity in their relationships (Beck et al. 1998). The ways in which they succeed might better inform our understanding of how different species can evolve to form higher-level individuals.

\section{Conclusion}

The recent development of experimental systems for studying the origins of multicellularity have shaped our understanding of an important major transition in evolution. Yet, these systems do not capture a class of evolutionary routes to multicellularity that are characterized by the gradual evolution of increased interdependence between initially free-living organisms. Lichens and microbial syntrophies highlight diverse routes to egalitarian multicellularity and shed light on the main challenges and possible ways to overcome them. In this regard, lichens, with their unique combination of phylogenetic diversity and extensive life history variation, would make exemplar model systems to extract general principles. Thus, ongoing efforts to better understand lichen evolutionary and natural history may pay unexpected dividends in our understanding of how non-canonical multicellular organisms arise.

Author ORCID. (DD Eric Libby, 0000-0002-6569-5793.

\section{References}

Ariew A and Lewontin RC (2004) The confusions of fitness. British Journal for the Philosophy of Science 55, 347-363.

Armaleo D and Clerc P (1991) Lichen chimeras: DNA analysis suggests that one fungus forms two morphotypes. Experimental Mycology 15, 1-10.

Bantinaki E, Kassen R, Knight CG, Robinson Z, Spiers AJ and Rainey PB (2007) Adaptive divergence in experimental populations of Pseudomonas fluorescens. III. Mutational origins of wrinkly spreader diversity. Genetics 176, 441-453.

Beck A, Friedl T and Rambold G (1998) Selectivity of photobiont choice in a defined lichen community: inferences from cultural and molecular studies. New Phytologist 139, 709-720.

Bonner JT (2009) First Signals: The Evolution of Multicellular Development. Princeton, New Jersey: Princeton University Press.
Boraas ME, Seale DB and Boxhorn JE (1998) Phagotrophy by a flagellate selects for colonial prey: a possible origin of multicellularity. Evolutionary Ecology 12, 153-164.

Bowler P and Rundel P (1975) Reproductive strategies in lichens. Botanical Journal of the Linnean Society 70, 325-340.

Cremer J, Melbinger A and Frey E (2012) Growth dynamics and the evolution of cooperation in microbial populations. Scientific Reports 2, 281.

de la Torre R, Sancho LG, Horneck G, de los Ríos A, Wierzchos J, Olsson-Francis K, Cockell CS, Rettberg P, Berger T, de Vera J-PP, et al. (2010) Survival of lichens and bacteria exposed to outer space conditions - results of the Lithopanspermia experiments. Icarus 208, 735-748.

D'Souza G and Kost C (2016) Experimental evolution of metabolic dependency in bacteria. PLoS Genetics 12, e1006364.

D’Souza G, Shitut S, Preussger D, Yousif G, Waschina S and Kost C (2018) Ecology and evolution of metabolic cross-feeding interactions in bacteria. Natural Product Reports 35, 455-488.

Dunne WM (2002) Bacterial adhesion: seen any good biofilms lately? Clinical Microbiology Reviews 15, 155-166.

Estrela S, Kerr B and Morris JJ (2016) Transitions in individuality through symbiosis. Current Opinion in Microbiology 31, 191-198.

Gallie J, Libby E, Bertels F, Remigi P, Jendresen CB, Ferguson GC, Desprat N, Buffing MF, Sauer U, Beaumont HJ, et al. (2015) Bistability in a metabolic network underpins the de novo evolution of colony switching in Pseudomonas fluorescens. PLoS Biology 13, e1002109.

Gargas A, DePriest PT, Grube M and Tehler A (1995) Multiple origins of lichen symbioses in fungi suggested by SSU rDNA phylogeny. Science 268, 1492-1495.

Gavrilets S (2010) Rapid transition towards the division of labor via evolution of developmental plasticity. PLoS Computational Biology 6, e1000805.

Godfrey-Smith P (2009) Darwinian Populations and Natural Selection. Oxford: Oxford University Press.

Grosberg RK and Strathmann RR (2007) The evolution of multicellularity: a minor major transition? Annual Review of Ecology, Evolution, and Systematics 38, 621-654.

Grube M and Berg G (2009) Microbial consortia of bacteria and fungi with focus on the lichen symbiosis. Fungal Biology Reviews 23, 72-85.

Hawksworth D (1988) The variety of fungal-algal symbioses, their evolutionary significance, and the nature of lichens. Botanical Journal of the Linnean Society 96, 3-20.

Herron MD, Ratcliff WC, Boswell J and Rosenzweig F (2018) Genetics of a de novo origin of undifferentiated multicellularity. Royal Society Open Science 5(8), 180912.

Herron MD, Borin JM, Boswell JC, Walker J, Chen I-CK, Knox CA, Boyd M, Rosenzweig F and Ratcliff WC (2019) De novo origins of multicellularity in response to predation. Scientific Reports 9, 2328.

Hey J (2001) The mind of the species problem. Trends in Ecology and Evolution 16, 326-329.

Highsmith RC (1982) Reproduction by fragmentation in corals. Marine Ecology Progress Series 7, 207-226.

Hillesland KL, Lim S, Flowers JJ, Turkarslan S, Pinel N, Zane GM, Elliott N, Qin Y, Wu L, Baliga NS, et al. (2014) Erosion of functional independence early in the evolution of a microbial mutualism. Proceedings of the National Academy of Sciences of the United States of America 111, 14822-14827.

Hom EF and Murray AW (2014) Niche engineering demonstrates a latent capacity for fungal-algal mutualism. Science 345, 94-98.

Honegger R (1998) The lichen symbiosis - what is so spectacular about it? Lichenologist 30, 193-212.

Jacobeen S, Graba EC, Brandys CG, Day TC, Ratcliff WC and Yunker PJ (2018a) Geometry, packing, and evolutionary paths to increased multicellular size. Physical Review E 97, 050401.

Jacobeen S, Pentz JT, Graba EC, Brandys CG, Ratcliff WC and Yunker PJ (2018b) Cellular packing, mechanical stress and the evolution of multicellularity. Nature Physics 14, 286-290.

Kallus Y, Miller JH and Libby E (2017) Paradoxes in leaky microbial trade. Nature Communications 8, 1361.

King N (2004) The unicellular ancestry of animal development. Developmental Cell 7, 313-325. 
Klitgord N and Segrè D (2010) Environments that induce synthetic microbial ecosystems. PLoS Computational Biology 6, e1001002.

Knoll AH (2011) The multiple origins of complex multicellularity. Annual Review of Earth and Planetary Sciences 39, 217-239.

Kochin BF, Bull JJ and Antia R (2010) Parasite evolution and life history theory. PLoS Biology 8, e1000524.

Koschwanez JH, Foster KR and Murray AW (2011) Sucrose utilization in budding yeast as a model for the origin of undifferentiated multicellularity. PLoS Biology 9, e1001122.

Koza A, Moshynets O, Otten W and Spiers AJ (2011) Environmental modification and niche construction: developing $\mathrm{O}_{2}$ gradients drive the evolution of the wrinkly spreader. ISME Journal 5, 665-673.

Libby E and Rainey PB (2013a) A conceptual framework for the evolutionary origins of multicellularity. Physical Biology 10, 035001.

Libby E and Rainey PB (2013b) Eco-evolutionary feedback and the tuning of proto-developmental life cycles. PLoS ONE 8, e82274.

Libby E and Ratcliff WC (2014) Ratcheting the evolution of multicellularity. Science 346, 426-427.

Libby E, Ratcliff W, Travisano M and Kerr B (2014) Geometry shapes evolution of early multicellularity. PLoS Computational Biology 10, e1003803.

Libby E, Conlin PL, Kerr B and Ratcliff WC (2016) Stabilizing multicellularity through ratcheting. Philosophical Transactions of the Royal Society B: Biological Sciences 371, 20150444.

Libby E, Hébert-Dufresne L, Hosseini S-R and Wagner A (2019) Syntrophy emerges spontaneously in complex metabolic systems. PLoS Computational Biology 15, e1007169.

Lind PA, Farr AD and Rainey PB (2015) Experimental evolution reveals hidden diversity in evolutionary pathways. Elife 4, e07074.

Lutzoni F, Pagel M and Reeb V (2001) Major fungal lineages are derived from lichen symbiotic ancestors. Nature 411, 937-940.

Maynard Smith J and Szathmáry E (1995) The Major Transitions in Evolution. Oxford: Oxford University Press.

McDonald MJ, Gehrig SM, Meintjes PL, Zhang X-X and Rainey PB (2009) Adaptive divergence in experimental populations of Pseudomonas fluorescens. IV. Genetic constraints guide evolutionary trajectories in a parallel adaptive radiation. Genetics 183, 1041-1053.

Michod RE (2005) On the transfer of fitness from the cell to the multicellular organism. Biology and Philosophy 20, 967-987.

Morris BEL, Henneberger R, Huber H and Moissl-Eichinger C (2013) Microbial syntrophy: interaction for the common good. FEMS Microbiology Reviews 37, 384-406.

Morris JJ, Lenski RE and Zinser ER (2012) The black queen hypothesis: evolution of dependencies through adaptive gene loss. mBio 3, e00036-12.

Nadell CD, Drescher K and Foster KR (2016) Spatial structure, cooperation and competition in biofilms. Nature Reviews Microbiology 14, 589-600.

Nagy LG, Kovács GM and Krizsán K (2018) Complex multicellularity in fungi: evolutionary convergence, single origin, or both? Biological Reviews 93, 1778-1794.

Nash TH, III (2008) Lichen Biology, 2nd edition. Cambridge: Cambridge University Press.

Okasha S (2006) Evolution and the Levels of Selection. Oxford: Oxford University Press.

Oliveira NM, Niehus R and Foster KR (2014) Evolutionary limits to cooperation in microbial communities. Proceedings of the National Academy of Sciences of the United States of America 111, 17941-17946.

Pande S, Merker H, Bohl K, Reichelt M, Schuster S, de Figueiredo LF, Kaleta C and Kost C (2014) Fitness and stability of obligate cross-feeding interactions that emerge upon gene loss in bacteria. ISME Journal 8, 953-962.

Poullain V, Gandon S, Brockhurst MA, Buckling A and Hochberg ME (2008) The evolution of specificity in evolving and coevolving antagonistic interactions between a bacteria and its phage. Evolution 62, 1-11.

Queller DC (1997) Cooperators since life began. Quarterly Review of Biology 72, 184-188.
Queller DC and Strassmann JE (2009) Beyond society: the evolution of organismality. Philosophical Transactions of the Royal Society B: Biological Sciences 364, 3143-3155.

Queller DC and Strassmann JE (2016) Problems of multi-species organisms: endosymbionts to holobionts. Biology and Philosophy 31, 855-873.

Rainey PB and Rainey K (2003) Evolution of cooperation and conflict in experimental bacterial populations. Nature 425, 72-74.

Rainey PB and Travisano M (1998) Adaptive radiation in a heterogeneous environment. Nature 394, 69-72.

Ratcliff WC, Denison RF, Borrello M and Travisano M (2012) Experimental evolution of multicellularity. Proceedings of the National Academy of Sciences of the United States of America 109, 1595-1600.

Ratcliff WC, Herron MD, Howell K, Pentz JT, Rosenzweig F and Travisano M (2013) Experimental evolution of an alternating uni- and multicellular life cycle in Chlamydomonas reinhardtii. Nature Communications 4, 2742.

Ratcliff WC, Fankhauser JD, Rogers DW, Greig D and Travisano M (2015) Origins of multicellular evolvability in snowflake yeast. Nature Communications 6, 6102.

Ratcliff WC, Herron M, Conlin PL and Libby E (2017) Nascent life cycles and the emergence of higher-level individuality. Philosophical Transactions of the Royal Society B: Biological Sciences 372, 20160420.

Richardson DH (1999) War in the world of lichens: parasitism and symbiosis as exemplified by lichens and lichenicolous fungi. Mycological Research 103, 641-650.

Roze D and Michod RE (2001) Mutation, multilevel selection, and the evolution of propagule size during the origin of multicellularity. American Naturalist 158, 638-654.

Schink B (2005) Syntrophic associations in methanogenic degradation. In Overmann J (ed.), Molecular Basis of Symbiosis. Berlin, Heidelberg: Springer, pp. 1-19.

Shapiro JW and Turner PE (2018) Evolution of mutualism from parasitism in experimental virus populations. Evolution 72(3), 707-712.

Smukalla S, Caldara M, Pochet N, Beauvais A, Guadagnini S, Yan C, Vinces MD, Jansen A, Prevost MC, Latgé J-P, et al. (2008) FLO1 is a variable green beard gene that drives biofilm-like cooperation in budding yeast. Cell 135, 726-737.

Spribille T, Tuovinen V, Resl P, Vanderpool D, Wolinski H, Aime MC, Schneider K, Stabentheiner E, Toome-Heller M, Thor G, et al. (2016) Basidiomycete yeasts in the cortex of ascomycete macrolichens. Science 353, 488-492.

Stams AJ and Plugge CM (2009) Electron transfer in syntrophic communities of anaerobic bacteria and archaea. Nature Reviews Microbiology 7, 568-577.

Suga H, Chen Z, de Mendoza A, Sebé-Pedrós A, Brown MW, Kramer E, Carr M, Kerner P, Vervoort M, Sánchez-Pons N, et al. (2013) The Capsaspora genome reveals a complex unicellular prehistory of animals. Nature Communications 4, 2325.

Szathmáry E (2015) Toward major evolutionary transitions theory 2.0. Proceedings of the National Academy of Sciences of the United States of America 112, 10104-10111.

Tan J, He Q, Pentz JT, Peng C, Yang X, Tsai M-H, Chen Y, Ratcliff WC and Jiang L (2019) Copper oxide nanoparticles promote the evolution of multicellularity in yeast. Nanotoxicology 13, 597-605.

Tarnita CE, Taubes CH and Nowak MA (2013) Evolutionary construction by staying together and coming together. Journal of Theoretical Biology 320, 10-22.

Tuovinen V, Ekman S, Thor G, Vanderpool D, Spribille T and Johannesson H (2019) Two basidiomycete fungi in the cortex of wolf lichens. Current Biology 29, 476-483.

Willensdorfer M (2009) On the evolution of differentiated multicellularity. Evolution 63, 306-323.

Zaremba-Niedzwiedzka K, Caceres EF, Saw JH, Bäckström D, Juzokaite L, Vancaester E, Seitz KW, Anantharaman K, Starnawski P, Kjeldsen KU, et al. (2017) Asgard archaea illuminate the origin of eukaryotic cellular complexity. Nature 541, 353-358. 